\title{
Simple Estimation of Förster Resonance Energy Transfer (FRET) Orientation Factor Distribution in Membranes
}

\author{
Luís M. S. Loura ${ }^{1,2}$
}

1 Faculty of Pharmacy, University of Coimbra, Health Sciences Campus, Azinhaga de Santa Comba, 3000-548 Coimbra, Portugal; E-Mail: 1loura@ff.uc.pt; Tel.: +351-239-488-485;

Fax: +351-239-827-126

2 Centre for Chemistry-Coimbra, Rua Larga, 3004-535 Coimbra, Portugal

Received: 20 September 2012; in revised form: 7 November 2012 / Accepted: 13 November 2012 / Published: 19 November 2012

\begin{abstract}
Because of its acute sensitivity to distance in the nanometer scale, Förster resonance energy transfer (FRET) has found a large variety of applications in many fields of chemistry, physics, and biology. One important issue regarding the correct usage of FRET is its dependence on the donor-acceptor relative orientation, expressed as the orientation factor $\kappa^{2}$. Different donor/acceptor conformations can lead to $\kappa^{2}$ values in the $0 \leq \kappa^{2} \leq 4$ range. Because the characteristic distance for FRET, $R_{0}$, is proportional to $\left(\kappa^{2}\right)^{1 / 6}$, uncertainties in the orientation factor are reflected in the quality of information that can be retrieved from a FRET experiment. In most cases, the average value of $\kappa^{2}$ corresponding to the dynamic isotropic limit $\left(<\kappa^{2}>=2 / 3\right)$ is used for computation of $R_{0}$ and hence donor-acceptor distances and acceptor concentrations. However, this can lead to significant error in unfavorable cases. This issue is more critical in membrane systems, because of their intrinsically anisotropic nature and their reduced fluidity in comparison to most common solvents. Here, a simple numerical simulation method for estimation of the probability density function of $\kappa^{2}$ for membrane-embedded donor and acceptor fluorophores in the dynamic regime is presented. In the simplest form, the proposed procedure uses as input the most probable orientations of the donor and acceptor transition dipoles, obtained by experimental (including linear dichroism) or theoretical (such as molecular dynamics simulation) techniques. Optionally, information about the widths of the donor and/or acceptor angular distributions may be incorporated. The methodology is illustrated for special limiting cases and common membrane FRET pairs.
\end{abstract}


Keywords: fluorescence; FRET; kappa-squared; linear dichroism; lipid bilayer; membrane probe; molecular dynamics

\section{Introduction}

Fluorescence spectroscopy has been utilized as a major tool in biophysics in general, and membrane studies in particular, for decades. Several parameters can be measured in steady-state (quantum yield, anisotropy) or time-resolved (intensity and anisotropy decays) conditions, and it allows monitoring of photophysical processes that are sensitive to distance, concentration (Förster Resonance Energy Transfer, FRET), aggregation (FRET, static quenching) or diffusion (collisional quenching). Fluorescence's versatility and sensitivity have earned it a place among the most useful biophysical techniques [1]. In particular, FRET is a most useful approach to the investigation of diverse problems in membrane biophysics, including membrane protein mapping, lateral heterogeneity (membrane domains), determination of the transverse location (depth) of fluorescent residues/labels inside the membrane, protein/lipid selectivity (preference of a specific lipid for the protein vicinity), and membrane protein oligomerization [2], both in spectroscopic studies and, more recently, under the microscope [3].

Additionally, quantitative applications of membrane fluorescence studies to the recovery of structural and partition information frequently require, as input, results obtained from other techniques. Some of these can be measured experimentally, such as area/lipid, lipid molar volume or bilayer thickness. However, one parameter for which there is no experimental technique suited to a definite measurement (though it was shown by Dale and coworkers that intervals containing it can be inferred from adequate fluorescence anisotropy measurents [4]) is the FRET orientation factor, $\kappa^{2}$. Because the characteristic distance for FRET, $R_{0}$, is proportional to $\left(\kappa^{2}\right)^{1 / 6}$, uncertainties in the orientation factor are reflected on the quality of information that can be retrieved from a FRET experiment. Most often, the theoretical value for the so-called dynamic isotropic limit $\left(\left\langle\kappa^{2}>=2 / 3\right)\right.$ is used, but $\left\langle\kappa^{2}>\right.$ uncertainty is still widely regarded as an inconvenience [5] that may be especially important in membranes, because of their intrinsic anisotropic nature and the restricted rotational mobility experienced by fluorophores incorporated inside the bilayer. The $2 / 3$ value corresponds to the dynamic limit, where rotation of both donor and acceptor is fast compared with the excited state lifetime.

As described in detail below in Section 3.1., the instant value of $\kappa^{2}$ for a given donor-acceptor pair (which falls in the $0 \leq \kappa^{2} \leq 4$ range) can be calculated from adequate molecular frame vectors. Although this definition of $\kappa^{2}$ is not suited to experimental measurement, it can be conveniently used in molecular simulation studies. In particular, from the instant position coordinates of a molecular dynamics (MD) simulation trajectory, calculation of the orientation factor for a given FRET donor-acceptor molecular pair is straightforward, and averaging both over pairs and over time is conveniently carried out. This has been used in a number of literature reports (e.g., [6-9]) including membrane-embedded fluorophores [10]. However, this approach is not a choice for most experimental researchers, as setup and validation of MD simulations for novel fluorophores (for which parameterization is seldom available) is not trivial. 
Even though computational calculations of $\kappa^{2}$ are scarce, determination of membrane-embedded fluorophore orientations using computational (for reviews of MD studies of fluorescent membrane probes, see $[11,12]$ ) or experimental techniques (traditionally linear dichroism or other spectroscopic methods $[13,14]$, or, more recently, methods based on polarized total internal reflection fluorescence microscopy or other microscopic techniques [15-17]) are more frequent.

This manuscript describes a simple manner to use data on probe location and orientation or alignment in membranes, obtained by experimental or molecular simulation methods, to provide estimates for the $\kappa^{2}$ distribution in the dynamic limit, from numerical simulation. For this purpose, a simple program was developed to generate random planar location distributions of donor and acceptor probes. Fluorophore orientation is then generated for each donor or acceptor taking into account information provided by the user. Subsequently, $\kappa^{2}$ is calculated for a large number of donor/acceptor pairs, and hence the $\kappa^{2}$ distribution and average value are obtained. Average $\kappa^{2}$ values for common membrane FRET pairs were obtained and are shown. For other FRET applications, the reader may use the software, which is available as supporting information to this article, without difficulty. This simplified approach enables immediate estimation of $\kappa^{2}$ distributions for quantitative FRET applications, without the need to resort to sophisticated theoretical or experimental techniques.

\section{Results and Discussion}

\subsection{Special Theoretical Cases}

This manuscript describes a simple manner to use data on probe orientation or alignment in membranes to estimate the distribution of the $\kappa^{2}$ FRET factor, using a small program described in more detail in Section 3. In this subsection we use the program to verify some results known from the literature, as well as to investigate other hypothetical scenarios of interest.

\subsubsection{Isotropic Dipole Distribution}

As described in Section 3, the software assumes normal distributions of $\cos \theta_{\mathrm{D}}$ and $\cos \theta_{\mathrm{A}}$ (truncated to the range $-1 \leq \cos \theta \leq 1$ ), where $\theta_{\mathrm{D}}$ and $\theta_{\mathrm{A}}$ are the tilts of donor and acceptor (respectively) relative to the normal to the membrane plane. The angles corresponding to the distribution maxima are read, as well as the standard deviations $\sigma_{\mathrm{A}}$ and $\sigma_{\mathrm{B}}$. If very wide angular distributions are used $(\sigma>>1)$ uniform azimuth angular distributions $f(\theta)=\sin \theta / 2$ are in practice obtained, as illustrated in Figure 1A. Therefore, and because $\varphi$ distribution is uniform in the $[0,2 \pi]$ interval, isotropic orientation of both donor and acceptor is easily simulated.

Figure $1 \mathrm{~B}$ shows the resulting probability density of $\kappa^{2}$ compared with the analytical result for isotropic dipoles, which is (e.g., [5]):

$$
p\left(\kappa^{2}\right)= \begin{cases}\frac{1}{2 \sqrt{3 \kappa^{2}}} \ln (2+\sqrt{3}) & 0 \leq \kappa^{2} \leq 1 \\ \frac{1}{2 \sqrt{3 \kappa^{2}}} \ln \left(\frac{2+\sqrt{3}}{\sqrt{\kappa^{2}}+\sqrt{\kappa^{2}-1}}\right) & 1 \leq \kappa^{2} \leq 4\end{cases}
$$


Figure 1. (a) Comparison of the simulated donor (red) and acceptor (green) density probabilities of orientation for planar distribution with the uniform distribution, $\sin \theta / 2$ (black); (b) Comparison of the simulated $\kappa^{2}$ density function (blue) with the theoretical curve of Equation 1 (black). $\left\langle\kappa^{2}\right\rangle=0.66$. Inputs: coplanar distribution of donor and acceptor, $\theta_{\mathrm{D}, \mathrm{MAX}}=\theta_{\mathrm{A}, \mathrm{MAX}}=90^{\circ}, \sigma_{\mathrm{A}}=\sigma_{\mathrm{B}}=3 ; N=10^{7}$ donor-acceptor pairs.
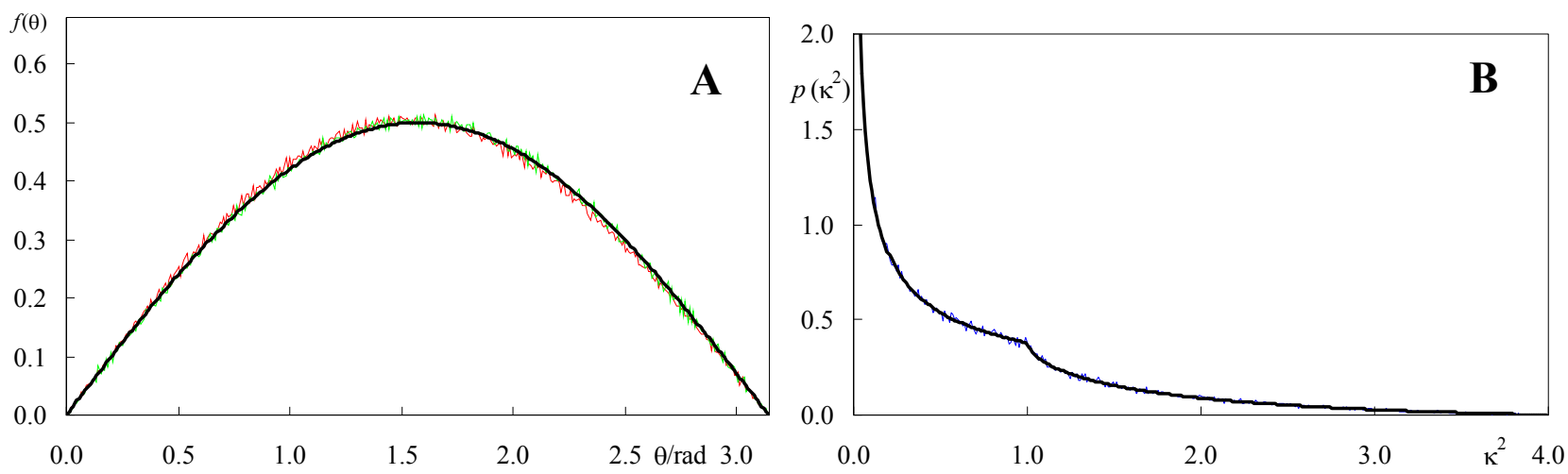

From the density distribution, one can calculate the average value,

$$
\left\langle\kappa^{2}\right\rangle=\int_{0}^{4} \kappa^{2} p\left(\kappa^{2}\right) d \kappa^{2}=2 / 3
$$

As shown in Figure 1B, there is excellent agreement between both simulated and analytical distributions and average $\kappa^{2}$ value. The result $\left\langle\kappa^{2}\right\rangle=2 / 3$, described in the literature [18] for planar probe distribution, is identical to that for isotropic orientation of dipoles distributed in a three dimensional system. The latter can also be obtained from the program, with a slight modification for the position of the acceptor in each pair (allowing a randomly allocated acceptor $z$ value - varying e.g., between 0 and a maximal value specified by the user - in each donor-acceptor pair). Again, there is excellent agreement with the theoretical expectations (not shown).

\subsubsection{Identical or Identically-Distributed Fluorophores}

This situation is illustrated in the inset of Figure 2. In this case, the distribution and average value of $\kappa^{2}$ for planar systems depend solely on the angle $\theta_{\mathrm{D}}$ between the donor transition dipole and the normal to the membrane plane. An analytical solution to this situation was described by Knoester and van Himbergen [19], who showed that

$$
\left\langle\kappa^{2}\right\rangle=\frac{2}{3}-\frac{2}{3} S+S^{2}
$$

where

$$
S=\frac{3\left\langle\cos ^{2} \theta_{D}\right\rangle-1}{2}
$$

is an orientation order parameter, equal to the average value of the second-order Legendre polynomial $P_{2}(\theta)$. For this particular case, since no orientation heterogeneity is (as yet) assumed, $\left.<\cos \theta_{\mathrm{D}}\right\rangle=\cos \theta_{\mathrm{D}}$. 
This result, which arguably is little known in the membrane fluorescence research community, is however very pertinent e.g., for FRET among identical fluorophores (energy migration, energy transfer or homo-FRET).

Figure 2 shows that the variation of $\left\langle\kappa^{2}>\right.$ against $\theta_{\mathrm{D}}$, obtained using our numerical approach, agrees with the analytical result. Large relative variations are observed, and between local maxima for $\theta_{\mathrm{D}}=0$ $\left(<\kappa^{2}>=\kappa^{2}(\right.$ invariant $\left.)=1\right), 90^{\circ}\left(<\kappa^{2}>=1.25\right)$ and $180^{\circ}\left(<\kappa^{2}>=\kappa^{2}(\right.$ invariant $\left.)=1\right)$, there are minima $\left(<\kappa^{2}>=5 / 9\right)$ at $\theta_{\mathrm{D}}=\operatorname{acos}\left((5 / 9)^{1 / 2}\right)=41.81^{\circ}$ and $180^{\circ}-41.81^{\circ}=138.19^{\circ}$. There are four $\theta_{\mathrm{D}}$ values for which an average of $2 / 3$ is obtained. These values correspond to the angles in the $\left[0,180^{\circ}\right]$ interval for which $S=0 \Leftrightarrow \cos \theta_{\mathrm{D}}= \pm(1 / 3)^{1 / 2}$ (the so-called magic angle 54.74 ${ }^{\circ}$, and $125.26^{\circ}$ ) and $S=2 / 3 \Leftrightarrow \cos \theta_{\mathrm{D}}= \pm(7 / 9)^{1 / 2}\left(28.13^{\circ}, 151.87^{\circ}\right)$. However, despite $<\kappa^{2}>$ being identical to the isotropic value, the $\kappa^{2}$ distributions are quite different. As shown in Figure 3 , the $\kappa^{2}$ distribution for $\theta_{\mathrm{D}}=54.74^{\circ}$ shows peaks at $\kappa^{2} \approx 0.12$ and $\kappa^{2} \approx 1$ (absent in the isotropic distribution), while it vanishes abruptly for $\kappa^{2}>25 / 9=2.778$. This latter higher limiting value corresponds to the situation of coplanar donor dipole, acceptor dipole and separation vector, with the two dipoles facing each other. On the contrary, in the isotropic limit, values of $\kappa^{2}$ near 4 (corresponding to collinear dipoles) are allowed and no cutoff is observed.

Figure 2. Average value of the orientation factor, $\left\langle\kappa^{2}\right\rangle$, as a function of the angle $\theta_{D}$, between the fluorophore transition and the normal dipole to the membrane plane, for two-dimensional FRET between donors and acceptors with identical orientation (see inset for illustration). Calculations with $\theta_{\mathrm{D}}$ values spaced by $7.5^{\circ}$ were carried out to obtain the points (the error bars length corresponds to two standard deviations of ten short simulations performed with $N=20,000$ donor/acceptor pairs). The solid line is the analytical solution obtained by Knoester and van Himbergen [19].

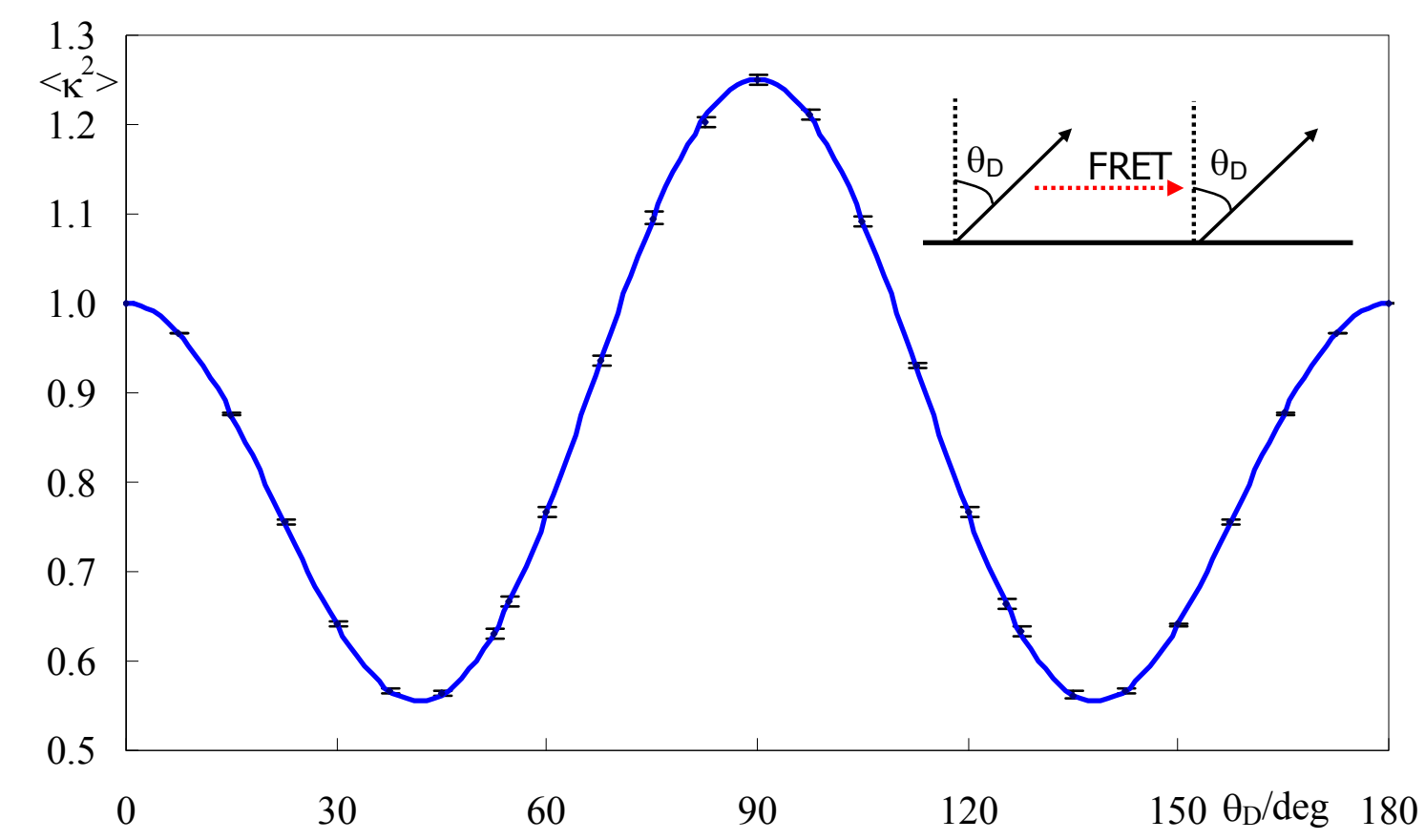


Figure 3. Comparison of the simulated $\kappa^{2}$ density function for $\theta_{D}=\theta_{A}=54.74^{\circ}$ (blue) with the isotropic theoretical curve of Equation 1 (black).

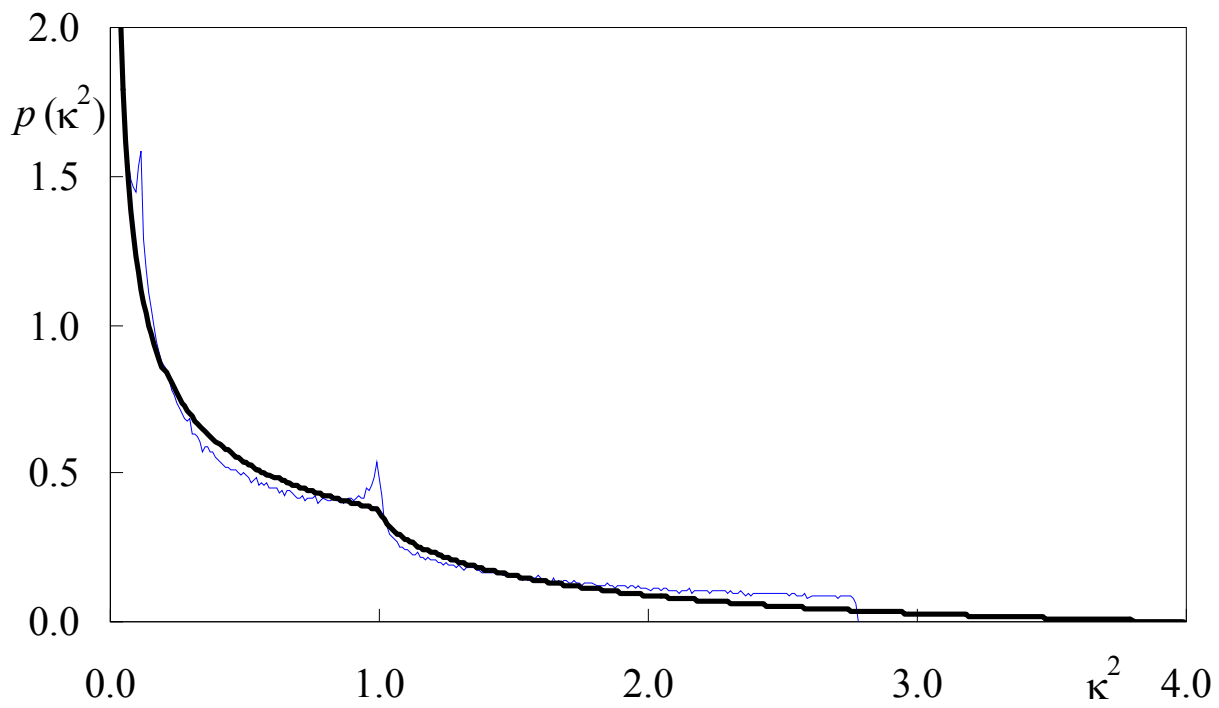

To illustrate the utility of this curve for quick estimation of $\left\langle\kappa^{2}\right\rangle$, we consider the recent MD study of 1-palmitoyl,2-[12-amino]dodecanoyl-sn-glycero-3-phosphocholine (C12-NBD-PC) in a fluid bilayer of 1,2-dipalmitoyl-sn-3-glycerophoshocholine (DPPC, $T=323 \mathrm{~K}$ ). The angular distribution of the transition dipole has a maximum around $\sim 120-125^{\circ}$ relative to the bilayer normal [20]. From Figure 2, this would be consistent with $\left\langle\kappa^{2}\right\rangle=0.67-0.77$. Direct calculation of $\kappa^{2}$ using the atomic coordinates of the MD trajectory leads accordingly to $\left\langle\kappa^{2}\right\rangle=0.72 \pm 0.04$ for molecules in the same-leaflet [10].

\subsection{Effect of Orientation Heterogeneity}

One important feature of our numerical procedure for estimation of $\kappa^{2}$ distributions of membrane-embedded fluorophores is that it allows the introduction of orientation heterogeneity, in the form of a normal distribution of $\cos \theta_{\mathrm{D}}$ and/or $\cos \theta_{\mathrm{A}}$, as mentioned previously. This type of heterogeneity is expected in simple rotational models such as the "wobbling-in-cone" motions [21]. The standard deviations of the $\cos \theta_{\mathrm{D}}$ and $\cos \theta_{\mathrm{A}}$ distributions $\left(\sigma_{\mathrm{D}}\right.$ and $\sigma_{\mathrm{A}}$, respectively) are a measure of the extent of freedom of dipole orientation. Therefore, they serve a similar purpose to that of Dale et al.'s depolarization factors $\left\langle d_{\mathrm{D}}{ }^{\mathrm{x}}>\right.$ and $\left\langle d_{\mathrm{A}}{ }^{\mathrm{x}}\right\rangle$ [4]: $\sigma$ and $\left\langle d^{\mathrm{x}}>\right.$ are different ways to express the orientation distributions sampled by donor and acceptor. Whereas Dale et al.'s form of taking this orientation redistribution into account using depolarization factors was justified by their perceived possibility of estimation of $\left\langle\kappa^{2}\right\rangle$ ranges from time-resolved fluorescence polarization measurements, $\sigma$ is a technique-independent, more direct metric of the dipole orientation heterogeneity.

Intuitively, upon permitting sampling of different donor/acceptor relative orientations, $\kappa^{2}$ distributions and average values are necessarily affected. As discussed above, the limiting case of $\sigma \gg>1$ leads to the expected isotropic case. Here we address the more physical possibility of moderate heterogeneity $(\sigma \approx 0.05-0.3)$ and its effect upon the orientation factor. Figures 4 and 5 illustrate the alterations produced on $\kappa^{2}$ distributions and average values upon widening the angular distributions of donor and acceptor dipoles for two distinct situations: identical donor and acceptor distributions (e.g., 
homo-FRET) centered around $\theta=90^{\circ}$ (Figure 4) and quite distinct donor and acceptor distributions centered around $60^{\circ}$ and $120^{\circ}$, respectively (Figure 5). In these calculations, a larger number of pairs (at least $10^{6}$ ) should be considered, to allow a better sampling of the $\theta_{\mathrm{D}}$ and $\theta_{\mathrm{A}}$ distributions. In both cases, it can be seen that whereas moderate orientation heterogeneity produces visible alterations in $\kappa^{2}$ distribution, significant effects require a very large degree of heterogeneity. This is more apparent for the system where donor and acceptor have very distinct angular distributions (Figure 5), where, remarkably, very wide distributions of both probes affect $\kappa^{2}$ by a degree $<10 \%$.

Figure 4. Effects of widening donor and acceptor dipole angular distributions (identical, centered around $90^{\circ}$, depicted in (a)) on the simulated $\kappa^{2}$ density functions (b); Colors indicate standard deviations of $\cos \theta$ distributions as follows: black, $\sigma=0$; blue, $\sigma=0.05$; red, $\sigma=0.10$; green, $\sigma=0.20$; magenta, $\sigma=0.30$. The numbers given in the inset of $(\mathbf{b})$ correspond to the average $\kappa^{2}$ value. $N=10^{6}$ donor-acceptor pairs.
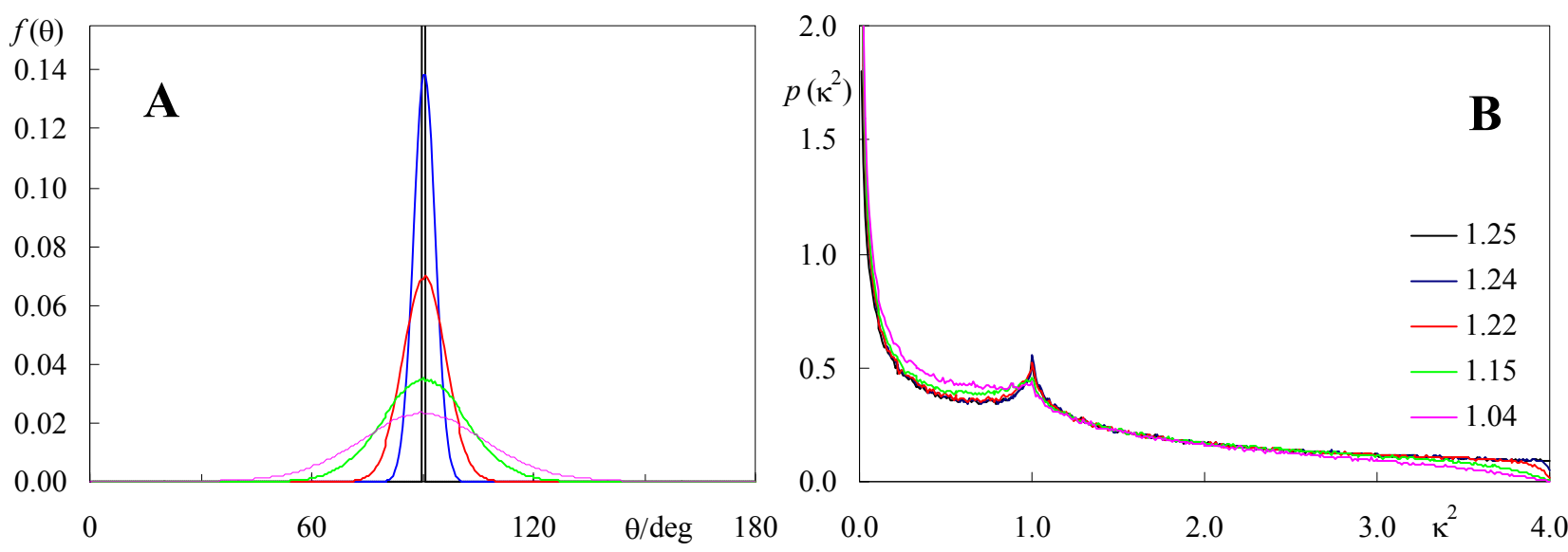

Figure 5. Effects of widening donor and acceptor dipole angular distributions (distinct, centered around $60^{\circ}$ (donor, solid curves) and $120^{\circ}$ (acceptor, dotted curves), depicted in (a)) on the simulated $\kappa^{2}$ density functions (b); See Figure 4 for color scheme. The numbers given in the inset of (b) correspond to the average $\kappa^{2}$ value. $N=10^{6}$ donor-acceptor pairs.
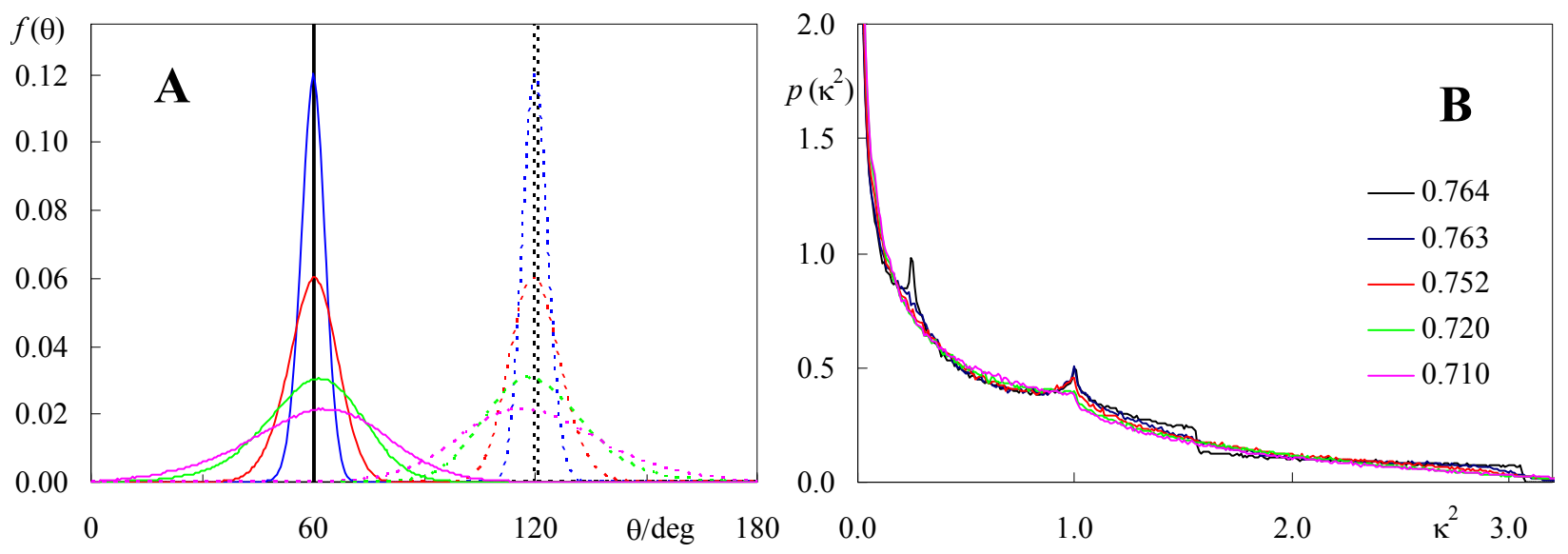


\subsection{Distance Effects}

In most applications of intramolecular FRET (involving a single acceptor fluorophore as quencher of each donor), distance measurements are carried out assuming a single fixed value of $R_{0}$, assuming a $\left\langle\kappa^{2}\right\rangle$ value (usually 2/3) independent of donor-acceptor distance. However, this view has been challenged, as MD simulations revealed considerable correlation between $\kappa^{2}$ and donor-acceptor distance in a labeled protein [6], possibly related to constraints imposed by the linker used to attach the donor probe. Naturally, in systems where each donor may transfer its excitation energy to multiple acceptors at varying distances, correlation between donor-acceptor distance and their relative orientation will necessarily occur if the dynamic isotropic limit is not met. Consider for example that donors and acceptors are located in separate parallel planes (different depths in the membrane), and that both types of fluorophores have narrow orientation distribution. The range and relative weight of possible $\kappa^{2}$ values will certainly differ for a donor-acceptor pair in which the acceptor is located directly below or above the donor, relative to the case where their lateral separation is large. Therefore, the question is not whether there exists correlation, but to which extent $\kappa^{2}$ may vary as a function of distance, and how to best take this into account.

For the sake of illustration, we consider the same donor and acceptor orientations as in the previous subsection, but assuming fixed (no orientation heterogeneity) $\theta_{\mathrm{D}}=\theta_{\mathrm{A}}=90^{\circ}$ and $\left(\theta_{\mathrm{D}}, \theta_{\mathrm{A}}\right)=\left(60^{\circ}, 120^{\circ}\right)$. We also consider two separate possibilities in each case: (i) coplanar donor and acceptor spatial distributions; (ii) donor and acceptor planes separated by an arbitrary distance of $h=2.0 \mathrm{~nm}$. In each of these scenarios, the size of the square systems $l$ was varied up to $1000 \mathrm{~nm} .10^{6}$ pairs were simulated for proper sampling of donor/acceptor distances. It is clear from the figure that location of both donor and acceptor dipoles in the same plane always lead to identical distributions (Figure 6A,E) and average values (Figure 6B,F). At variance, for donors and acceptors placed in parallel planes, there are very significant changes in both $\kappa^{2}$ distribution (Figure $6 \mathrm{C}, \mathrm{G}$ ) and $\left\langle\kappa^{2}\right\rangle$ (Figure $6 \mathrm{D}, \mathrm{H}$ ), when the size of the simulated system (which constraints the maximal possible donor-acceptor distance) is $<<100 \mathrm{~nm}$.

In this case, definition of an average $\kappa^{2}$ becomes a problem. The best way to deal with this situation is to take notice of how FRET occurs from a given donor (with fluorescence lifetime in absence of acceptor equal to $\tau_{0}$ ) to a distribution of $M$ acceptors (where the distance between the selected donor and the ith-acceptor is denoted by $R_{\mathrm{i}}$ ). The decay rate $k$ is given by

$$
k=\tau_{0}^{-1}+k_{T}=\tau_{0}^{-1}+\sum_{i=1}^{M} k_{i}
$$

where the different individual FRET rates are calculated according to

$$
k_{i}=\frac{1}{\tau_{0}}\left(\frac{R_{0_{i}}}{R_{i}}\right)^{6}
$$

Finally, the Förster radius for each donor-acceptor pair is calculated using

$$
R_{0 i}=0.02108\left[\kappa_{i}^{2} \Phi_{0} n^{-4} \int_{0}^{\infty} \lambda^{4} I(\lambda) \varepsilon(\lambda) d \lambda\right]^{1 / 6}
$$


Figure 6. $\kappa^{2}$ distributions $(\mathbf{a}, \mathbf{c}, \mathbf{e}, \mathbf{g})$ and average values $(\mathbf{b}, \mathbf{d}, \mathbf{f}, \mathbf{h})$ obtained for identical (centered around $90^{\circ} ;(\mathbf{a}-\mathbf{d})$ ) and distinct (centered around $60^{\circ}$ (donor) and $120^{\circ}$ (acceptor); (g-h)) fluorophore dipole distributions, both for coplanar donors and acceptors $(\mathbf{a}-\mathbf{b}, \mathbf{e}-\mathbf{f})$ and with parallel donor and acceptor planes (separated by $h=2.0 \mathrm{~nm},(\mathbf{c}-\mathbf{d}$, $\mathbf{g}-\mathbf{h})$ ), for varying simulated system size, $l$. Colors in the left panels indicate the value of $l$ (black, $1 \mathrm{~nm}$; blue, $2 \mathrm{~nm}$; red, $4 \mathrm{~nm}$; green, $8 \mathrm{~nm}$; magenta, $100 \mathrm{~nm}$ ). All lines in panels (a) and (e) are essentially identical.
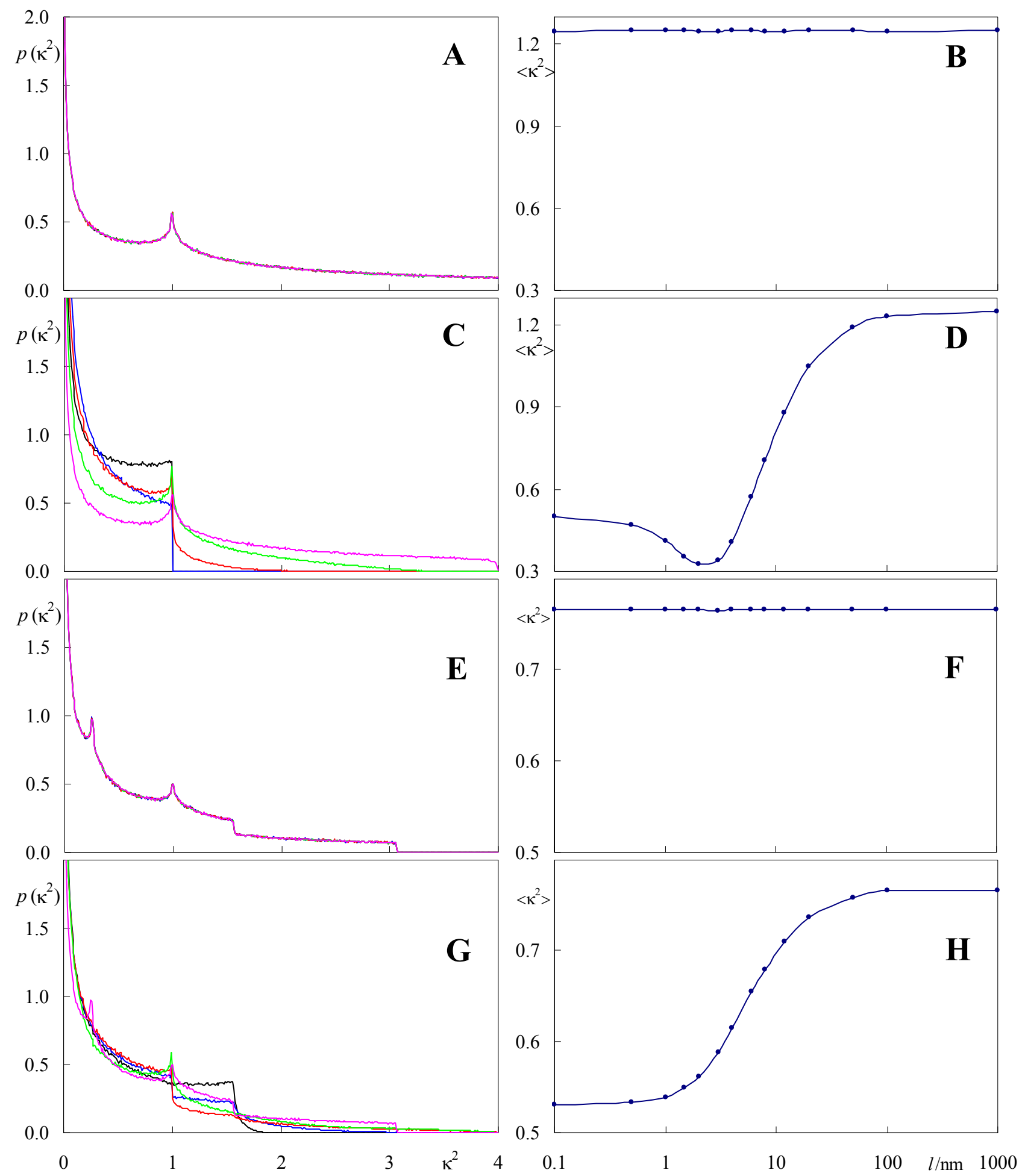
where $\Phi_{0}$ is the quantum yield of the donor in the absence of acceptor molecules, $n$ is the refractive index of the medium, and the integral term is the overlap between the normalized donor emission $(I(\lambda))$ and the acceptor absorption $(\varepsilon(\lambda))$ spectra. The constant value in the equation above assumes nm units for both $\lambda$ and $R_{0}$, as well as $\mathrm{M}^{-1} \mathrm{~cm}^{-1}$ units for $\varepsilon(\lambda)$. Combining these three equations, one concludes that the FRET rate is given by

$$
k_{T}=C \sum_{i=1}^{M} \frac{\kappa_{i}^{2}}{R_{i}^{6}}
$$

where $C$ is a constant term for all acceptors in the distribution. That is, the natural way to average $\kappa^{2}$ over different acceptors, with different distances to a given donor, is to use the inverse sixth power of the donor-acceptor distance as weight. In this way, acceptors located more closely contribute significantly more to the average (as they are also responsible for most of the quenching by FRET). For the purpose of this calculation, we adapted our program for averaging $\kappa^{2}$ in this way for FRET between a plane of donors and another of acceptors, and FRET in a bilayer geometry (with possibility of FRET between a plane of donors and two planes of acceptors - one in each bilayer leaflet). We then proceeded to calculate $\left\langle\kappa^{2}>\right.$ for the two angular distributions presented above $\left(\theta_{\mathrm{D}}=\theta_{\mathrm{A}}=90^{\circ}\right.$; and $\left.\left(\theta_{\mathrm{D}}, \theta_{\mathrm{A}}\right)=\left(60^{\circ}, 120^{\circ}\right)\right)$ assuming a bilayer geometry with $h_{1}=0.0$ and $h_{2}=2.0 \mathrm{~nm}$ as the donor-acceptor interplanar distances. For coplanar fluorophores, an exclusion distance of $R_{\mathrm{e}}=0.8 \mathrm{~nm}$ was also introduced, as donors and acceptors in the same plane cannot lie closer than a given minimum distance value. This would be similar to a typical experiment, with acceptor distribution in both bilayer leaflets. For a better sampling of all possible orientations for small $R_{\mathrm{i}}$ values, a value of $l=100 \mathrm{~nm}$ was used. Ten different simulations were carried out in each case, to assess whether proper convergence was obtained. For $\theta_{\mathrm{D}}=\theta_{\mathrm{A}}=90^{\circ}$, an average of 1.237 with standard deviation 0.010 was obtained, whereas for $\left(\theta_{\mathrm{D}}, \theta_{\mathrm{A}}\right)=\left(60^{\circ}, 120^{\circ}\right)$ the corresponding values were 0.771 and 0.009 . The relatively small standard deviations indicate that sampling was adequate. The average values were similar (very slightly lower) to the $\left\langle\kappa^{2}\right\rangle$ calculated for the planar systems (1.250 and 0.776 , respectively), and the small differences are due to the contribution of acceptors lying in the opposite leaflet. Because of the transverse distance of $2 \mathrm{~nm}$, neglecting this leaflet does not lead to considerable error. In any case, for the following section that applies this methodology to computation of $\left\langle\kappa^{2}>\right.$ for common experimental donor-acceptor pairs, whenever possible, information on donor-acceptor interplanar distances was taken into account.

\subsection{Application to Experimental FRET Pairs}

Table 1 summarizes relevant information regarding membrane probes that are commonly involved in FRET experiments (as donors, acceptors or both), Förster pair combinations formed by these probes, examples of quantitative experimental FRET studies using these pairs, and information obtained from literature simulation or experimental studies regarding fluorophore location and transition dipole orientation of the probes. The latter information was used to calculate an average $\kappa^{2}$ value using the inverse sixth power of the donor-acceptor distance as weight, as described in the previous subsection. These averages are also shown in the Table. It can be seen that all these values but one (homo-FRET between Rhodamine B probes) lie within $15 \%$ or 0.1 of the isotropic limit of $2 / 3$, 
even though the probe orientation distributions are quite varied. The most probable explanation for this is that all orientation data retrieved from the literature refer to liquid disordered bilayers, in which probes have generally broad angular distributions. Applications to FRET in liquid ordered or gel phases, where conformational freedom is largely reduced, would potentially lead to $\left\langle\kappa^{2}>\right.$ further from the $2 / 3$ value. This is illustrated in the $t$-PnA/DPH pair. For $t$-PnA, contrary to other probes, information regarding orientation distribution is also available in the gel phase $\left(5^{\circ}, 0.03\right.$ (gel) [22]). Using these input values instead of the fluid phase parameters, one would obtain $\left\langle\kappa^{2}\right\rangle=0.53 \pm 0.01$ instead of $0.58 \pm 0.01$ for both probes in the fluid phase. It should also be emphasized that the dipole orientation details shown in Table 1 refer to a specific probe compound labelled with the fluorophore under consideration, and other probes exist where the same fluorophore could be expected to show different transverse location and/or orientation distribution in the bilayer. For example, NBD data are based in a MD study of NBD-PC, whereas FRET studies also employ other NBD probes (NBD-PE, NBD-cholesterol).

\subsection{Effect of $<\kappa^{2}>$ on FRET Efficiency}

The most common experimental observables in FRET are the decay of donor in the presence of acceptor, $i_{\mathrm{DA}}(t)$, and the FRET efficency, $E$. The decay law $i_{\mathrm{DA}}(t)$ for bilayer geometries and uniform probe distribution is available in the literature [23]

$$
\begin{aligned}
& i_{D A}(t)=\exp \left(-\frac{t}{\tau_{0}}\right) \exp \left\{-\pi R_{0}^{2} c \gamma\left[\frac{2}{3},\left(\frac{R_{0}}{R_{e}}\right)^{6}\left(\frac{t}{\tau_{0}}\right)\right]\left(\frac{t}{\tau_{0}}\right)^{1 / 3}\right\} . \\
& \cdot \exp \left\{\pi R_{e}^{2} c\left(1-\exp \left[-\left(\frac{R_{0}}{R_{e}}\right)^{6}\left(\frac{t}{\tau_{0}}\right)\right]\right)\right\}
\end{aligned}
$$

In this equation, $c$ is the number of acceptors per unit area, $\gamma$ is the incomplete gamma function, and the other symbols have the same meaning as in the previous equations. Although Equation 7 was originally derived for a plane of acceptors containing the donor (cis transfer), it is also valid if the donor molecule is separated from the acceptor plane by a distance $R_{\mathrm{e}}$. From the time-resolved donor emission, the FRET efficiency, which is defined (and often experimentally measured) by

$$
E=1-I_{D A} / I_{D}
$$

(where $I_{\mathrm{DA}}$ and $I_{\mathrm{D}}$ are the donor steady-state emission intensities in presence and absence of donor, respectively), can be calculated using

$$
E=1-\int_{0}^{\infty} i_{D A}(t) d t / \int_{0}^{\infty} i_{D}(t) d t
$$

In the latter equation, $i_{\mathrm{D}}(t)=\exp \left(-t / \tau_{0}\right)$ is the donor time-resolved fluorescence decay in the absence of acceptor.

To appreciate the effect of $\left\langle\kappa^{2}>\right.$ on the FRET efficiency, we first define $\overline{R_{0}}$ as the value of the Förster radius calculated with $\kappa^{2}=2 / 3$, and then consider a form of Equation 7 in terms of 
dimensionless parameters $\zeta=t / \tau_{0}$ (reduced time), $\sigma=\pi \overline{R_{0}}{ }^{2} c$ (number of acceptors in a circle of radius equal to $\left.\overline{R_{0}}\right), \alpha=\overline{R_{0}} / R_{\mathrm{e}}$ and $\beta=(3 / 2)<\kappa^{2}>$ :

$$
i_{D A}(\varsigma)=\exp (-\varsigma) \exp \left\{-\beta^{1 / 3} \sigma\left(\gamma\left[\frac{2}{3}, \beta \alpha^{6} \varsigma\right] \varsigma^{1 / 3}-\frac{1-\exp \left(-\beta \alpha^{6} \varsigma\right)}{\beta^{1 / 3} \alpha^{2}}\right)\right\}
$$

This form of the decay law is suitable for calculation (by integration over $\zeta$, done using Equation 9 after substitution of variables $t$ and $\zeta$ ) of universal curves $E v s$. $\sigma$, for fixed values of $\alpha$ and $\beta$. Representative examples are shown in Figure 7.

Figure 7. FRET efficiency $E$ as a function of dimensionless acceptor concentration $\sigma=\pi \bar{R}_{0}^{2} c$, for given values of $\alpha=\overline{R_{0}} / R_{\mathrm{e}}$ (panels $((\mathbf{a}-\mathbf{d})$ correspond to $\alpha=0.5,1.0,2.0$, and 5.0, respectively) and $\left\langle\kappa^{2}>\right.$ (in each panel, curves obtained with $\left\langle\kappa^{2}\right\rangle=0.30,0.50,2 / 3$, 0.80 and 1.25 are show in blue, red, black, magenta and green, respectively).
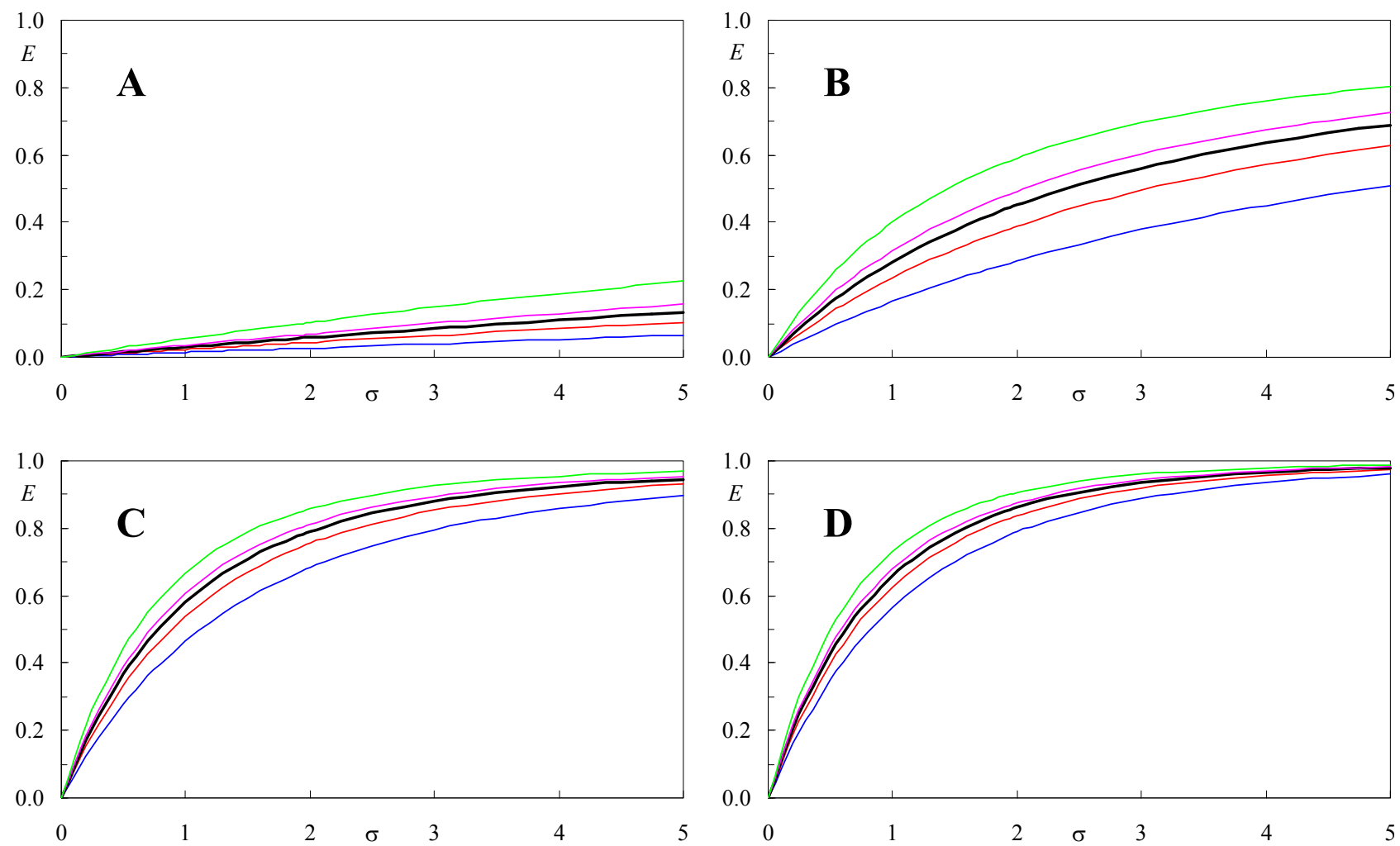

From Figure 7, it can be seen that variations in FRET efficiency upon changing the $<\kappa^{2}>$ value used to calculate $R_{0}$ are most important when the latter is smaller than or comparable to the exclusion distance, such as in panels (a) and (b). For example, for $\alpha=\overline{R_{0}} / R_{\mathrm{e}}=0.50$, the FRET efficiency obtained for $\left\langle\kappa^{2}\right\rangle=1.25$ is $>3$ times larger than that obtained for for $\left\langle\kappa^{2}>=0.30\right.$ over the whole $\sigma<5$ range. However, it must be noted that larger values of $\alpha=\overline{R_{0}} / R_{\mathrm{e}}$ are often met in membranes, as long as $R_{0}$ has a reasonable $(\sim 2.5 \mathrm{~nm}$ or larger) value and there are acceptors in the same bilayer leaflet as the donors. In these cases, such as illustrated in panels (c) and (d), the effect of $\left\langle\kappa^{2}\right\rangle$ on $E$, though still clearly detectable, is smaller, especially in the $0.50<\left\langle\kappa^{2}\right\rangle<0.80$ range which is observed for most common FRET pairs (Table 1). 
Table 1. Donor and acceptor location and orientation literature data for common FRET pairs in membranes, and $<\kappa^{2}>$ calculated from this information.

\begin{tabular}{|c|c|c|c|c|c|c|c|}
\hline $\begin{array}{c}\text { Donor } \\
\text { fluorophore }^{\mathrm{a}}\end{array}$ & $\begin{array}{c}\text { Acceptor } \\
\text { fluorophore }\end{array}$ & $\begin{array}{l}\text { Quantitative } \\
\text { experimental } \\
\text { FRET studies }\end{array}$ & $\begin{array}{c}\text { Donor transverse } \\
\text { location }^{b}\end{array}$ & $\begin{array}{l}\text { Donor orientation } \\
\qquad\left(\theta_{\max }, \sigma\right)^{c}\end{array}$ & $\begin{array}{c}\text { Acceptor transverse } \\
\text { location }\end{array}$ & $\begin{array}{l}\text { Acceptor orientation } \\
\qquad\left(\theta_{\max }, \sigma\right)^{\mathrm{c}}\end{array}$ & $\begin{array}{l}\text { Calculated } \\
<\kappa^{2}>d\end{array}$ \\
\hline$t$-PnA & DPH & {$[24,25]$} & $0.92 \mathrm{~nm}[26]^{\mathrm{e}}$ & $20^{\circ}, 0.15^{\mathrm{f}}$ & $0.75 \mathrm{~nm}[27]^{\mathrm{f}}$ & $0,0.35[27]^{\mathrm{f}}$ & $0.58 \pm 0.01$ \\
\hline DPH & NBD & [28-31] & $0.75 \mathrm{~nm}[27]^{\mathrm{f}}$ & $0,0.35[27]^{\mathrm{f}}$ & $1.35 \mathrm{~nm}[20,32,33]^{\mathrm{e}, \mathrm{f}}$ & $125^{\circ}, 0.35[20]^{\mathrm{f}}$ & $0.66 \pm 0.01$ \\
\hline NBD & NBD & {$[34,35]$} & $1.35 \mathrm{~nm}[20,32,33]^{\mathrm{f}}$ & $125^{\circ}, 0.35[20]^{\mathrm{f}}$ & $1.35 \mathrm{~nm}[20,32,33]^{\mathrm{e}, \mathrm{f}}$ & & $0.66 \pm 0.03$ \\
\hline NBD & Carbocyanine & {$[36]$} & & & $1.26 \mathrm{~nm}[37]^{\mathrm{f}}$ & $77^{\circ}, 0.25[37]^{\mathrm{f}}$ & $0.76 \pm 0.02$ \\
\hline NBD & Rhodamine B & {$[25,35,38-42]$} & & & $2.1 \mathrm{~nm}[43,44]^{\mathrm{f}}$ & $90^{\circ}, 0.275[43,45]^{\mathrm{g}}$ & $0.56 \pm 0.02$ \\
\hline BODIPY & Rhodamine B & {$[30,46]$} & $0.8 \mathrm{~nm}[47]^{\mathrm{f}}$ & $10^{\circ}, 0.5[47]^{\mathrm{f}}$ & & & $0.57 \pm 0.01$ \\
\hline BODIPY & Carbocyanine & [48] & & & $1.26 \mathrm{~nm}[37]^{\mathrm{f}}$ & $77^{\circ}, 0.25[37]^{\mathrm{f}}$ & $0.72 \pm 0.02$ \\
\hline Rhodamine B & Rhodamine B & {$[25,35,41,49,50]$} & $2.1 \mathrm{~nm}[43,44]^{\mathrm{f}}$ & $90^{\circ}, 0.275[43,45]^{\mathrm{g}}$ & $2.1 \mathrm{~nm}[43,44]^{\mathrm{f}}$ & $90^{\circ}, 0.275[43,45]^{\mathrm{g}}$ & $1.08 \pm 0.01$ \\
\hline
\end{tabular}

${ }^{\mathrm{a}} t$-PnA: trans-parinaric acid; DPH: diphenylhexatriene; NBD: 7-nitrobenz-2-oxa-1,3-diazol-4-yl)amino; BODIPY: 4,4-difluoro-3a,4adiaza-s-indacene; ${ }^{\mathrm{b}}$ Relative to the center of the bilayer;

${ }^{\mathrm{c}}$ The values given refer to the $\theta_{\max }$ and $\sigma$ values (read by the simulation program) that produce angular distributions most consistent with the simulation or experimental data reported in the cited references; ${ }^{\mathrm{d}}$ Average and standard deviations of average for ten independent simulations with two planes, $R_{\mathrm{i}}^{-6}$ weight, $N=10^{6}, R_{\mathrm{e}}=0.8 \mathrm{~nm}$ and $l=100 \mathrm{~nm} ;{ }^{\mathrm{e}}$ Inferred from experimental fluorescence quenching data; ${ }^{\mathrm{f}}$ Inferred from simulation data; ${ }^{\mathrm{g}}$ Inferred from linear dichroism data. 


\section{Methods}

\subsection{Definition of $\kappa^{2}$ for a Given Donor-Acceptor Pair}

$\kappa^{2}$ is given by [5]

$$
\kappa^{2}=\left(\cos \psi_{T}-3 \cos \psi_{D} \cos \psi_{A}\right)^{2}
$$

where $\Psi_{\mathrm{T}}$ is the angle between the transition moments of the donor and acceptor and $\Psi_{\mathrm{D}}$ and $\Psi_{\mathrm{A}}$ are the angles between the donor and acceptor transition moments and the vector uniting their centers, $\vec{R}$ (Figure 8). An equivalent expression, involving scalar products of $\vec{r}=\vec{R} /\|\vec{R}\|$ and unit vectors $\vec{u}_{D}$ and $\vec{u}_{A}$ (in the direction of the donor and acceptor dipoles, respectively), is

$$
\kappa^{2}=\left(\vec{u}_{D} \cdot \vec{u}_{A}-3\left(\vec{r} \cdot \vec{u}_{D}\right)\left(\vec{r} \cdot \vec{u}_{A}\right)\right)^{2}
$$

Figure 8. Vectors and angles relevant to the orientation factor. Adapted from [10].

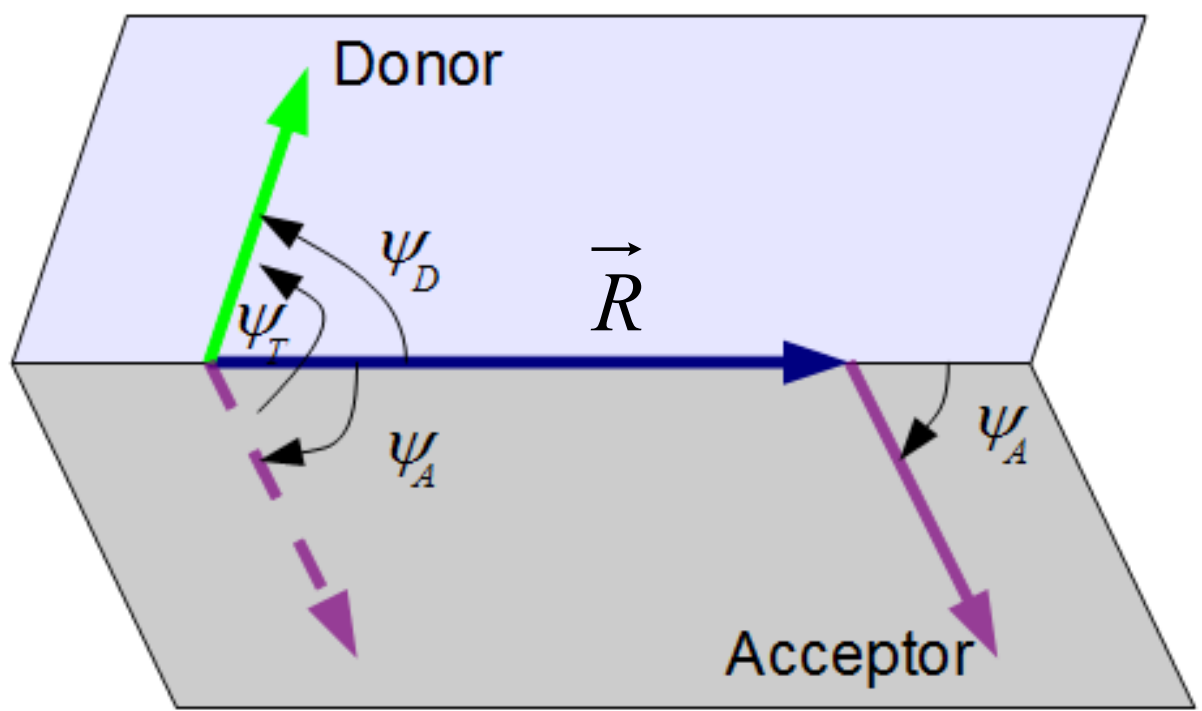

\subsection{Calculation of $\kappa^{2}$ Distributions and Averages from Numerical Simulation}

Two programs, written as macros in the Visual Basic Editor within a Microsoft Excel document, were developed for this article. The programs are available as supplemental files to this article, and may be edited by any user. Microsoft Excel Visual Basic was chosen as it may be easily used by most readers, and produces immediate numerical and graphic output.

Program 1 calculates $\kappa^{2}$ distributions and average values without weighting, for a single acceptor plane. The basic algorithm can be summarized as follows:

1. Read inputs: $\theta_{\mathrm{D}, \max }, \theta_{\mathrm{A}, \max }, \sigma_{\mathrm{D}}, \sigma_{\mathrm{A}}, l, N$, donor-acceptor interplanar distance $h$, and the number of bins (equal length subintervals of $0 \leq \kappa^{2} \leq 4$ ) for $\kappa^{2}$ distribution.

2. Main cycle - calculate $\kappa^{2}$ for the $i^{\text {th }}$ donor-acceptor pair:

2.1. Set donor and acceptor coordinates, $\left(x_{\mathrm{Di}}, y_{\mathrm{Di}}, z_{\mathrm{Di}}\right)$ and $\left(x_{\mathrm{Ai}}, y_{\mathrm{Ai}}, z_{\mathrm{Ai}}\right)$, respectively. For the $x$ and $y$-coordinates, uniformly distributed random numbers between 0 and $L$ are allotted. $z_{\mathrm{Di}}$ is taken as zero, whereas $z_{\mathrm{Ai}}$ is taken as $h$. 
2.2. Calculate donor-acceptor separating vector $\vec{R}$, and its modulus.

2.3. Set donor and acceptor dipole orientation coordinates, $\left(\theta_{\mathrm{Di}}, \varphi_{\mathrm{Di}}\right)$ and $\left(\theta_{\mathrm{Ai}}, \varphi_{\mathrm{Ai}}\right)$, respectively. For $\varphi_{\mathrm{Di}}$ and $\varphi_{\mathrm{Ai}}$, uniformly distributed random numbers between 0 and $2 \pi$ are allotted. $\theta_{\mathrm{Di}}$ and $\theta_{\mathrm{Ai}}$ (the angles relative to the bilayer normal, so that they fall between 0 and $\pi$ ) are calculated by firstly computing their cosines, using Gaussian random numbers centered around $\cos \theta_{\mathrm{D}, \max }$ and $\cos \theta_{\mathrm{A}, \max }$, with standard deviation $\sigma_{\mathrm{D}}$ and $\sigma_{\mathrm{A}}$, respectively. Gaussian random numbers are obtained using the Box-Muller transform method [51,52]. If either of the calculated cosines falls outside the $[-1,1]$ interval, new $\cos \theta_{\mathrm{Di}}$ and $\cos \theta_{\mathrm{Ai}}$ are calculated. Otherwise, $\theta_{\mathrm{Di}}$ and $\theta_{\mathrm{Ai}}$ are obtained in the $[0, \pi]$ interval by inversion.

2.4. Calculate Cartesian coordinates of unit dipole vectors $\vec{u}_{D}$ and $\vec{u}_{A}$, from $\left(\theta_{\mathrm{Di}}, \varphi_{\mathrm{Di}}\right)$ and $\left(\theta_{\mathrm{Ai}}, \varphi_{\mathrm{Ai}}\right)$.

2.5. Calculate $\kappa^{2}$ using Equation 12.

2.6. Update histogram of $\kappa^{2}$ distribution (in fact, the program also calculates $\theta_{\mathrm{D}}$ and $\theta_{\mathrm{A}}$ distributions, for testing and/or comparison and matching with distributions from the literature; these are also updated at this point), and partial sum of $\kappa^{2}$ values (for calculation of $\left\langle\kappa^{2}\right\rangle=\operatorname{sum}\left(\kappa^{2}{ }_{i}\right) / N$ at the end of the cycle).

2.7 Increment $\mathrm{i}$ and repeat until i $>N$.

3. Write outputs to the active worksheet: $\kappa^{2}$ average value and distribution, $\theta_{\mathrm{D}}$ and $\theta_{\mathrm{A}}$ distributions.

All calculations and distributions shown in Figures 1-6 were obtained with this program.

Program 2 follows a similar algorithm for calculation of $\kappa^{2}$ for the $i^{\text {th }}$ donor-acceptor pair. However, it computes an average $\kappa^{2}$ value using $R_{\mathrm{i}}^{-6}$ as weight for each pair. It may be used for both one and two acceptor planes. The program reads $\theta_{\mathrm{D}, \max }, \theta_{\mathrm{A}, \max }, \sigma_{\mathrm{D}}, \sigma_{\mathrm{A}}, l, N$, and, for both planes, the respective donor-acceptor interplanar $\left(h_{1}\right.$ and $\left.h_{2}\right)$ and exclusion $\left(R_{\mathrm{e} 1}\right.$ and $\left.R_{\mathrm{e} 2}\right)$ distances. Two main cycles are carried out, one for each acceptor plane, with calculation of $\kappa^{2}$ distribution in each case. For both cycles, calculation of $\kappa^{2}$ for the $i^{\text {th }}$ donor-acceptor pair is identical to that in the first program, with an additional check: if $R_{\mathrm{i}}<R_{\mathrm{e} 1}$ (first cycle) or $R_{\mathrm{i}}<R_{\mathrm{e} 2}$ (second cycle), then new donor and acceptor distance coordinates are allotted and a corresponding new $R_{\mathrm{i}}$ is calculated. Partial sums of $\kappa^{2} / R^{6}$ values are now computed, to obtain $<\kappa^{2}>=\operatorname{sum}\left(\kappa^{2}{ }_{\mathrm{i}} / R_{\mathrm{i}}{ }^{6}\right) / \operatorname{sum}\left(1 / R_{\mathrm{i}}{ }^{6}\right)$. As described in Section 3 , this weighting scheme reflects the dependence of the FRET interaction with distance, and was used to compute the values of the right column of Table 1 (see footnote for typical input parameters).

\section{Conclusions}

A simple method for calculation of the distribution and average value of the FRET orientation factor $\kappa^{2}$, for membrane-located donor and acceptor fluorophores, is presented. The method is implemented in two programs that are available with the present article. The programs use as inputs the donor and acceptor transverse locations, orientation and orientational heterogeneity. Analytical results previously obtained in limiting cases were verified, validating our approach. A number of donor/acceptor orientation distributions tested in this work produce $\kappa^{2}$ distributions and average values which are significantly different to that corresponding to the isotropic limit, characterized by $\left\langle\kappa^{2}\right\rangle=2 / 3$. Considerable dependence of $\left\langle\kappa^{2}>\right.$ on system size $l$ when the latter is $<<100 \mathrm{~nm}$ was verified, which presents a problem regarding the most appropriate $<\kappa^{2}>$ to consider for the purpose of 
quantitative analysis of FRET data. It is proposed that calculating $\left\langle\kappa^{2}\right\rangle$ using the sixth inverse power of the donor-acceptor distance is the most natural way to average $\kappa^{2}$ in this situation, and estimates of $<\kappa^{2}>$ were obtained for common membrane FRET pairs using available fluorophore location and orientation data. It was shown that for most of these systems, the calculated $<\kappa^{2}>$ lies within $\pm 15 \%$ of the $2 / 3$ value, possibly because probes in fluid bilayers have very broad orientation distributions. In these cases, substantial effects on FRET efficiency are not expected, provided that the Förster radius is significantly larger than the donor-acceptor exclusion distance. For more ordered bilayers, narrow orientation distributions may produce $<\kappa^{2}>$ further form the $2 / 3$ value. In these ordered systems, an additional problem is that fluorophore dipole orientation randomization may occur in a time scale of the order or slower than the donor excited state lifetime. In case reorientation proceeds much slower than transfer, the opposite regime (static limit) is operative. As discussed in detail elsewhere [4,5], in this limit, it is generally not possible to use $\kappa^{2}$ averages to calculate donor-acceptor distances in intramolecular FRET (the only exceptions occur for isotropic dipole distribution or when the FRET efficiency is vanishingly small). In the intermediate case of comparable rates of reorientation and transfer, FRET kinetics becomes very complex [19]. Because these situations of restricted mobility are not contemplated in our method, which calculates $\kappa^{2}$ distributions and averages corresponding to the dynamic limit, care must be taken when addressing these systems. However, even in such cases, the ease of application of this method - as long as fluorophore location and orientation data (or, at worst, "educated guesses") are available (in the literature or upon measurement) for the pair at hand-allows calculation of $\left\langle\kappa^{2}>\right.$ which will constitute an improvement over the widely used but very seldom justified $<\kappa^{2}>=2 / 3$ assumption.

\section{Acknowledgments}

The author acknowledges support by Fundação para a Ciência e Tecnologia (Portugal) through project PTDC/QUI-QUI/098198/2008, co-funded by FEDER (program FCOMP-01-0124FEDER-010787).

\section{References}

1. Lakowicz, J.R. Principles of Fluorescence Spectroscopy, 3rd ed.; Springer: New York, NY, USA, 2006.

2. Loura, L.M.S.; Prieto, M. FRET in membrane biophysics: An overview. Front Physiol. 2011, $2,82$.

3. De Almeida, R.F.; Loura, L.M.S.; Prieto, M. Membrane lipid domains and rafts: Current applications of fluorescence lifetime spectroscopy and imaging. Chem. Phys. Lipids 2009, 157, 61-77.

4. Dale, R.E.; Eisinger, J.; Blumberg, W.E. The orientational freedom of molecular probes. The orientation factor in intramolecular energy transfer. Biophys. J. 1979, 26, 161-193.

5. Van der Meer, B.W. Kappa-squared: From nuisance to new sense. Rev. Mol. Biotechnol. 2002, 82, 181-196.

6. VanBeek, D.B.; Zwier, M.C.; Shorb, J.M.; Krueger, B.P. Fretting about FRET: Correlation between $\kappa$ and R. Biophys. J. 2007, 92, 4168-4178. 
7. Allen, L.R.; Paci, E. Orientational averaging of dye molecules attached to proteins in Förster resonance energy transfer measurements: Insights from a simulation study. J. Chem. Phys. 2009, $131,065101$.

8. Hoefling, M.; Lima, N.; Haenni, D.; Seidel, C.A.M.; Schuler, B.; Grubmüller, H. Structural heterogeneity and quantitative FRET efficiency distributions of polyprolines through a hybrid atomistic simulation and Monte Carlo approach. PLoS One 2011, 6, e19791.

9. Deplazes, E.; Jayatilaka, D.; Corry, B. Testing the use of molecular dynamics to simulate fluorophore motions and FRET. Phys. Chem. Chem. Phys. 2011, 13, 11045-11054.

10. Loura, L.M.S.; Palace Carvalho, A.J.; Prates Ramalho, J.P. Direct calculation of Förster orientation factor of membrane probes by molecular simulation. J. Mol. Struct. THEOCHEM 2010, 946, 107-112.

11. Loura, L.M.S.; Prates Ramalho, J.P. Fluorescent membrane probes' behaviour in lipid bilayers: Insights from molecular dynamics simulations. Biophys. Rev. 2009, 1, 141-148.

12. Loura, L.M.S.; Prates Ramalho, J.P. Recent developments in molecular dynamics simulations of fluorescent membrane probes. Molecules 2011, 16, 5437-5452.

13. Hicks, M.R.; Kowałski, J.; Rodger, A. LD spectroscopy of natural and synthetic biomaterials. Chem. Soc. Rev. 2010, 39, 3380-3393.

14. Lopes, S.C.D.N.; Castanho, M.A.R.B. Overview of common spectroscopic methods to determine the orientation/alignment of membrane probes and drugs in lipidic bilayers. Curr. Org. Chem. 2005, 9, 889-898.

15. Oreopoulos, J.; Yip, C.M. Probing Membrane order and topography in supported lipid bilayers by combined polarized total internal reflection fluorescence-atomic force microscopy. Biophys. J. 2009, 96, 1970-1984.

16. Beausang, J.F.; Sun, Y.; Quinlan, M.E.; Forkey, J.N.; Goldman, Y.E. Orientation and Rotational Motions of Single Molecules by Polarized Total Internal Reflection Fluorescence Microscopy (polTIRFM). Cold Spring Harb. Protoc. 2012, doi:10.1101/pdb.top069344.

17. Corry, B; Jayatilaka, D.; Martinac, B.; Rigby, P. Determination of the orientational distribution and orientation factor for transfer between membrane-bound fluorophores using a confocal microscope. Biophys. J. 2006, 91, 1032-1045.

18. Baumann, J.; Fayer, M.D. Excitation transfer in disordered two-dimensional and anisotropic three-dimensional systems: Effects of spatial geometry on time-resolved observables. J. Chem. Phys. 1986, 85, 4087-4107.

19. Knoester, J.; van Himbergen, J.E. Effect of molecular reorientation on excitation decay due to incoherent energy transfer. J. Chem. Phys. 1984, 81, 4380-4388.

20. Loura, L.M.S.; Prates Ramalho, J.P. Location and dynamics of acyl chain NBD-labeled phosphatidylcholine (NBD-PC) in DPPC bilayers. A molecular dynamics and time-resolved fluorescence anisotropy study. Biochim. Biophys. Acta 2007, 1768, 467-478.

21. Kinosita, K.; Kawato, S.; Ikegami, A. A theory of fluorescence polarization decay in membranes. Biophys. J. 1977, 20, 289-305.

22. Fernandes, M.X.; García de la Torre, J.; Castanho, M.A.R.B. A brownian dynamics simulation of an acyl chain and a trans-parinaric acid molecule confined in a phospholipid bilayer in the gel and liquid-crystal phases. J. Phys. Chem. B 2000, 104, 11579-11584. 
23. Wolber, P.K.; Hudson, B.S. An analytic solution to the Förster energy transfer problem in two dimensions. Biophys. J. 1979, 28, 197-210.

24. Silva, L.; de Almeida, R.F.M.; Fedorov, A.; Matos, A.P.; Prieto, M. Ceramide-platform formation and -induced biophysical changes in a fluid phospholipid membrane. Mol. Membr. Biol. 2006, $23,137-148$.

25. Silva, L.C.; de Almeida, R.F.M.; Castro, B.M.; Fedorov, A.; Prieto, M. Formation of ceramide/sphingomyelin gel domains in the presence of an unsaturated phospholipid: A quantitative multiprobe approach. Biophys. J. 2007, 93, 1639-1650.

26. Fernandes, M.X.; García de la Torre, J.; Castanho, M.A.R.B. Joint determination by Brownian dynamics and fluorescence quenching of the in-depth location profile of biomolecules in membranes. Anal. Biochem. 2002, 307, 1-12.

27. Repáková, J.; Čapková, P.; Holopainen, J.M.; Vattulainen, I. Distribution, orientation, and dynamics of DPH probes in DPPC bilayer. J. Phys. Chem. B 2004, 108, 13438-13448.

28. Loura, L.M.S.; Coutinho, A.; Silva, A.; Fedorov, A.; Prieto, M. Structural effects of a basic peptide on the organization of dipalmitoylphosphatidylcholine/dipalmitoylphosphatidylserine membranes: A fluorescent resonance energy transfer study. J. Phys. Chem. B 2006, 110, 8130-8141.

29. Fernandes, F.; Loura, L.M.S.; Fedorov, A.; Prieto, M. Absence of clustering of phosphatidylinositol-(4,5)-bisphosphate in fluid phosphatidylcholine. J. Lipid Res. 2006, 47, 1521-1525.

30. Coutinho, A.; Loura, L.M.S.; Prieto, M. FRET studies of lipid-protein aggregates related to amyloid-like fibers. J. Neurochem. 2011, 116, 696-701.

31. Loura, L.M.S. Lateral distribution of NBD-PC fluorescent lipid analogs in membranes probed by molecular dynamics-assisted analysis of Förster Resonance Energy Transfer (FRET) and fluorescence quenching. Int. J. Mol. Sci. 2012, 13, 14545-14564.

32. Chattopadhyay, A.; London, E. Parallax method for direct measurement of membrane penetration depth utilizing fluorescence quenching by spin-labeled phospholipids. Biochemistry 1987, 26, 39-45.

33. Huster, D.; Müller, P.; Arnold, K.; Herrmann, A. Dynamics of membrane penetration of the fluorescent 7-nitrobenz-2-oxa-1,3-diazol-4-yl (NBD) group attached to an acyl chain of phosphatidylcholine. Biophys. J. 2001, 80, 822-831.

34. Prieto, M.J.E.; Castanho, M.; Coutinho, A.; Ortiz, A.; Aranda, F.J.; Gómez-Fernández, J.C. Fluorescence study of a derivatized diacylglycerol incorporated in model membranes. Chem. Phys. Lipids 1994, 69, 75-85.

35. Loura, L.M.S.; Fedorov, A.; Prieto, M. Membrane probe distribution heterogeneity: A resonance energy transfer study. J. Phys. Chem. B 2000, 104, 6920-6931.

36. Loura, L.M.S.; Fedorov, A.; Prieto, M. Partition of membrane probes in a gel/fluid two-component lipid system: A fluorescence resonance energy transfer study. Biochim. Biophys. Acta 2000, 1467, 101-112.

37. Gullapalli, R.R.; Demirel, M.C.; Butler, P.J. Molecular dynamics simulations of DiI-C ${ }_{18}(3)$ in a DPPC lipid bilayer. Phys. Chem. Chem. Phys. 2008, 10, 3548-3560.

38. Lantzsch, G.; Binder, H.; Heerklotz, H. Surface area per molecule in lipid/ $\mathrm{C}_{12} \mathrm{E}_{n}$ membranes as seen by fluorescence resonance energy transfer. J. Fluoresc. 1994, 4, 339-343. 
39. Loura, L.M.S.; Fedorov, A.; Prieto, M. Exclusion of a cholesterol analog from the cholesterol-rich phase in model membranes. Biochim. Biophys. Acta 2001, 1511, 236-243.

40. Loura, L.M.S.; Fedorov, A.; Prieto, M. Fluid-fluid membrane microheterogeneity: A fluorescence resonance energy transfer study. Biophys. J. 2001, 80, 776-788.

41. De Almeida, R.F.M.; Loura, L.M.S.; Fedorov, A.; Prieto, M. Lipid rafts have different sizes depending on membrane composition: A time-resolved fluorescence resonance energy transfer study. J. Mol. Biol. 2005, 346, 1109-1120.

42. Castro, B.M.; de Almeida, R.F.M.; Goormaghtigh, E.; Fedorov, A.; Prieto, M. Organization and dynamics of Fas transmembrane domain in raft membranes and modulation by ceramide. Biophys. J. 2011, 101, 1632-1641.

43. Medhage, B.; Mukhtar, E.; Kalman, B.; Johansson, L.B.-A.; Molotkovsky, J.G. Electronic energy transfer in anisotropic systems. Part 5-Rhodamine-lipid derivatives in model membranes. J. Chem. Soc. Faraday Trans. 1992, 88, 2845-2851.

44. Kyrychenko, A. A molecular dynamics model of rhodamine-labeled phospholipid incorporated into a lipid bilayer. Chem. Phys. Lett. 2010, 485, 95-99.

45. Johansson, L.B.-A.; Niemi, A. Electronic energy transfer in anisotropic systems. 1. Octadecylrhodamine B in vesicles. J. Phys. Chem. 1987, 91, 3020-3023.

46. Coutinho, A.; Loura, L.M.S.; Fedorov, A.; Prieto, M. Pinched multilamellar structure of aggregates of lysozyme and phosphatidylserine-containing membranes revealed by FRET. Biophys. J. 2008, 95, 4726-4736.

47. Song, K.C.; Livanec, P.W.; Klauda, J.B.; Kuczera, K.; Dunn, R.C.; Im, W. Orientation of fluorescent lipid analogue BODIPY-PC to probe lipid membrane properties: Insights from molecular dynamics simulations. J. Phys. Chem. B 2011, 115, 6157-6165.

48. Heberle, F.A.; Wu, J.; Goh, S.L.; Petruzielo, R.S.; Feigenson, G.W. Comparison of three ternary lipid bilayer mixtures: FRET and ESR reveal nanodomains. Biophys. J. 2010, 99, 3309-3318.

49. Loura, L.M.S.; Fedorov, A; Prieto, M. Resonance energy transfer in a model system of membranes: Application to gel and liquid crystalline phases. Biophys. J. 1996, 71, 1823-1836.

50. Castro, B.M.; de Almeida, R.F.M.; Fedorov, A.; Prieto, M. The photophysics of a Rhodamine head labeled phospholipid in the identification and characterization of membrane lipid phases. Chem. Phys. Lipids 2012, 165, 311-319.

51. Box, G.E.P.; Muller, M.E. A note on the generation of random normal deviates. Ann. Math. Stat. 1958, 29, 610-611.

52. Pike, M.C. Algorithm 267: Random normal deviate [G5]. Comm. ACM 1965, 8, 606.

(C) 2012 by the authors; licensee MDPI, Basel, Switzerland. This article is an open access article distributed under the terms and conditions of the Creative Commons Attribution license (http://creativecommons.org/licenses/by/3.0/). 